\title{
Téoros
}

Revue de recherche en tourisme

\section{La villégiature : un lien historique}

\section{Marcel Samson}

Volume 6, numéro 2, juillet 1987

Le Saint-Laurent magnétique

URI : https://id.erudit.org/iderudit/1080502ar

DOI : https://doi.org/10.7202/1080502ar

Aller au sommaire du numéro

Éditeur(s)

Université du Québec à Montréal

\section{ISSN}

0712-8657 (imprimé)

1923-2705 (numérique)

Découvrir la revue

Citer cet article

Samson, M. (1987). La villégiature : un lien historique. Téoros, 6(2), 6-8.

https://doi.org/10.7202/1080502ar d'utilisation que vous pouvez consulter en ligne.

https://apropos.erudit.org/fr/usagers/politique-dutilisation/ 


\title{
La villégiature: un lien historique
}

\author{
par Marcel Samson *
}

Par un certain nombre de documents, guides et récits auxquels vient s'ajouter récemment l'étude historique de Philippe Dubé sur la villégiature dans Charlevoix ${ }^{(1)}$, on sait maintenant que la villégiature privée a constitué un pilier, souvent oublié, de l'industrie touristique en devenir. Jusqu'au XVIII" siècle, à l'époque du Régime français, on retrace $1^{+}$existence de villas de campagne sur les bords du Saint-Laurent:

Un prélat français, amateur de belle nanure, Mgr Herman Dosquet, eveque de Samos in Partibus, dans ses visites pastorales, vers le commencement du siècle dernier, frappe sans doute par l'éclat du paysage, y fonda whe villa. Une plaque de métal, découverte dans les fondations, quand la maison fut rebâtie par $\mathrm{Mr}$. Sheppard, marque 1732 . (2)

Dans les environs de Québec particulièrement, la noblesse de cour et d'église avait donc adopté le même comportement que ses concitoyens de la mère-patrie. A cette différence qu'ils avaient un grand fleuve à leurs pieds. Mais c'était à cette époque, un phenomène encore très isolé; c'est avec l'arrivée de la société industrielle au XIX" siècle que la villégiature prend une certaine ampleur. C'est avec l'émergence de stations estivales dans la région du Bas-SaintLaurent qu'on a vu apparaittre les grandes villas de vacances. En même temps, se produit un mouvement semblable dans les environs de Québec et de Montréal. Mais, avant de parler plus spécifiquement des liens de la villćgiature et du Saint-Laurent, rappelons en quelques lignes à quoi ressemblait le Québec au milieu du XIX $\mathrm{X}^{*}$ siècle.

Selon les historiens Linteau, Durocher et Robert, c'est à partir de 1820 que le Québec a connu un débordement des paroisses vers les plateaux des Laurentides et des Cantons de $\mathrm{l}^{\prime} \mathrm{Est} \mathrm{t}^{(3)}$. Il faut retenir qu'au milieu du $\mathrm{XLX}^{\mathrm{e}}$ siècle, les Canadiensfrançais sont minoritaires à Montréal et ainsi que dans la ville de Québec où ils ne constituent que $60 \%$ de la population. C'est aussi vers 1850 que ces mémes historiens

"Marcel Sameon est professeur-chercheur a IrINRSUrbanisution. placent "tes débuts significatifs de l'industrialisation" ". En ce qui concerne I'urbanisation comme processus social, il faut également noter qu'en 1851 un peu moins de $15 \%$ des Québecois sont des urbains; plus du tiers le seront cinquante ans plus tard et c'est surtout durant la décennie 1871-1881 que ce phénomène s'est accéléré. Enfin, la structure sociale qu'on a pendant longtemps considérée comme extrểmement simple à la fin du XIX ${ }^{c}$ siècle était en fait plus complexe $e^{(4)}$. On a longtemps cru en effet que la société canadienne-française était formée de deux groupes, d'une part l'élite constituée essentiellement du clergé et des professions libérales et, d'autre part, la masse de la population composée majoritairement d'habitants confinés à l'agriculture. En fait, on peut distinguer une bourgeoisie composée de deux couches: la grande bourgeoisie qui possède presque tout le capital et contrôle les institutions économiques dominantes et la moyenne bourgeoisie qui dis- pose de moins de capital et qui exerce son contróle à un niveau intermédiaire. Il y a ensuite la petite bourgeoisie composée de professions liberales, de petits entrepreneurs, de marchands, qui est une classe intermédiaire entre la bourgeoisie et la masse des travailleurs et des paysans.

\section{Au $\mathrm{XIXe}$ siècle: \\ les grands centres de villégiature}

Considerant ce qu'on vient de dire sur le contexte socio-économique du Québec du milieu du $X I X^{e}$ siècle, il ne faut pas se surprendre que ce soit des classes privilégiées "etrangères", c'est-à-dire composies surtout d'Américains et de Canadiens anglophones appartenant à la grande bourgeoisie, qui créeront les premières stations balnéaires et montagnardes ${ }^{(5)}$.

Rappelons les principales caractéristiques de ces centres de villégiature.

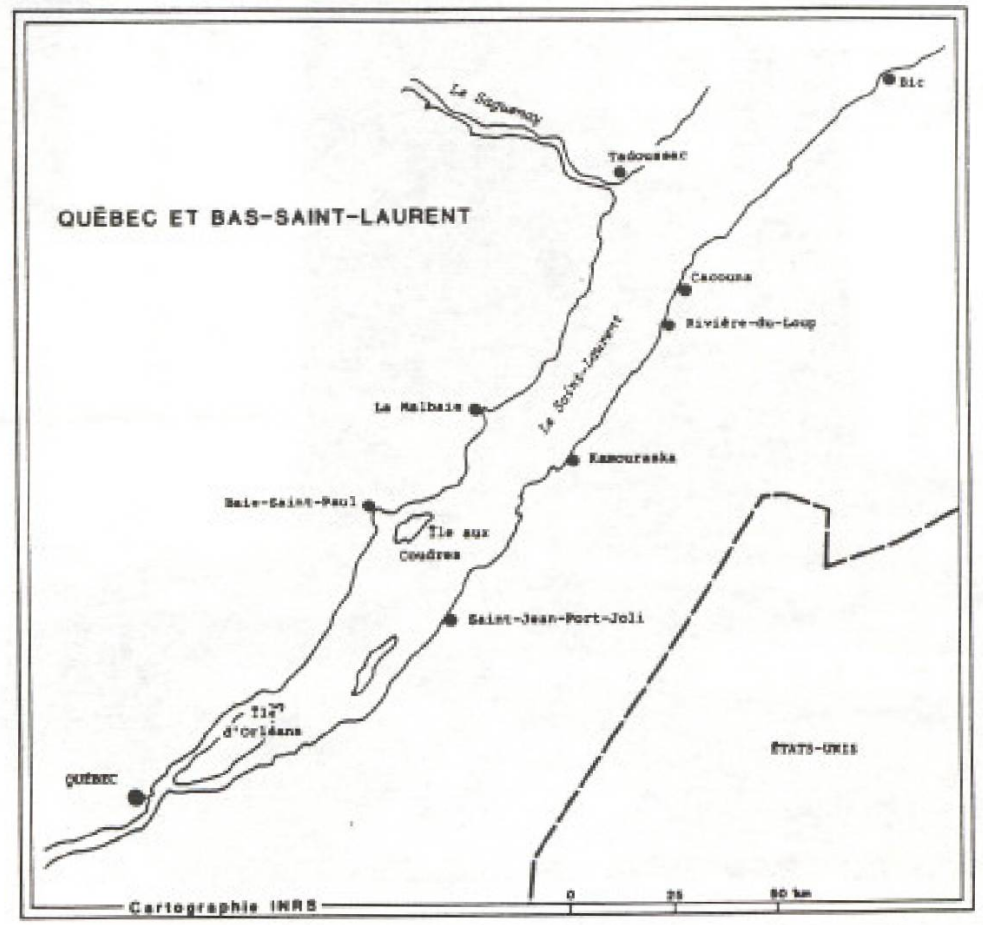




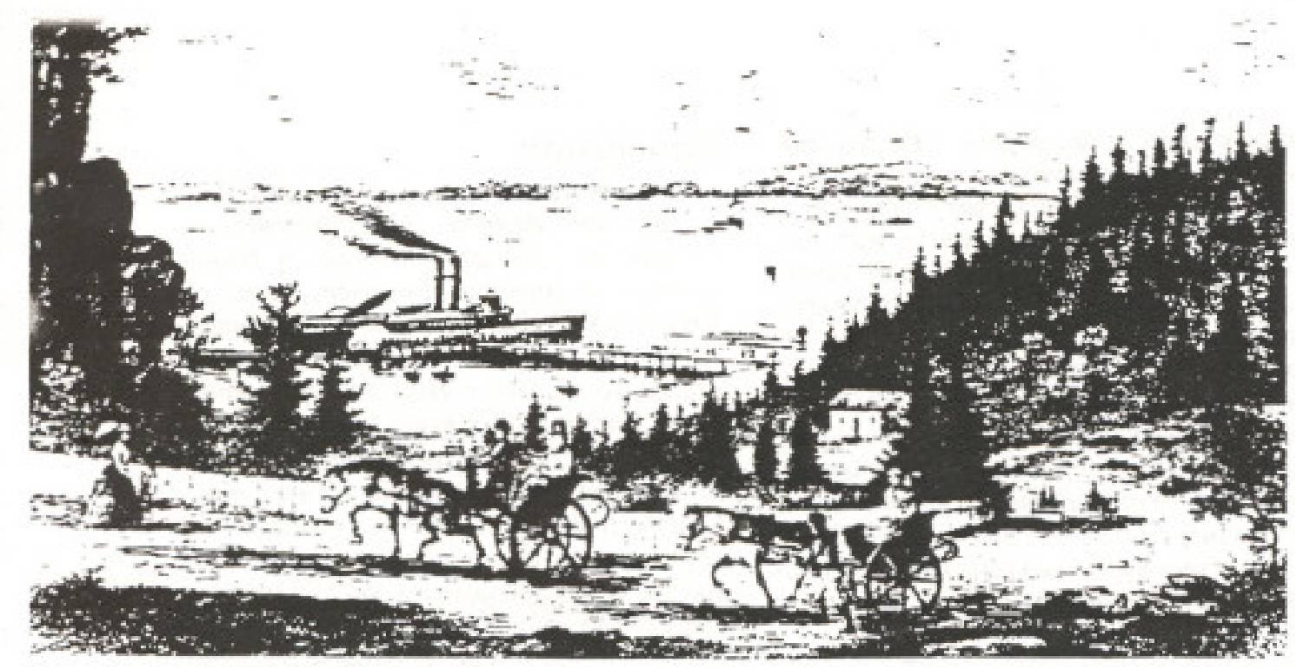

Le débarcadere at la Malbaie.

Il est intếressant d'abord de noter qu' 'avant mème le développement des grandes stations à la mode surtout fréquentées par la grande bourgeoisie anglaise et américaine, le petit village de Kamouraska en aval de Quebec, sur la rive sud du fleuve, fut prom bablement, selon le géographe Roger Brière, "la plus ancienne villégiature du Bas-du-Fleuve et probablement le plus ancien rendez-vous d'été du Québec" à laquelle on accédaît par goélette ou, par diligence, à partir de Lévis. Roger Brière rapporte les paroles d'Arthur Buies, grand chroniqueur et voyageur de la fin du $\mathrm{XIX}^{(5)}$ sibcle, qui affirmait que "les autres endroits ne comptaient pas"* et que le manoir du seigneur Taché (de la grande bourgeoisie politique de l'epoque) "avait reçu pendant un quart de siècle tout ce que le pays renfermait d'hommes éminents dans la vie publique, ou distingués par la naissance ou la position "ibs . Bref, au cours de la première moitié du XIXr siecle surtout, Kamouraska était le rendez-vous estival de "la crème des crèmes" de la société canadienne-française de l'époque. Ils étaient fort peu nombreux à faire partie de ce club sélect. Kamouraska eut donc son heure de gloire mais ce n'était encore que bien peu comparativement à ce que devaient connaitre quelques villages de la rive nord, de l'autre cóté du fleuve, avec l'arrivée de l'élite américaine et canadienne-anglaise. En 1853, avec le début des liaisons en bateau à vapeur avec les principaux villages en aval de Québec, et encore plus important, avec la présence en 1860 du chemin de fer, des centres de villégiature se développèrent et devinrent vite célèbres: Tadoussac, Métis-sur-mer, Cacouna et l'endroit le plus réputé d'entre tous, Murray Bay ou La Malbaie.

L'expansion des services fluviaux et des chemins de fer allaient rendre les attractions de l'estuaire du Saini-Laurent accessibles à une clientele de plus en plus nombreuse de Canadiens des deux Canada (Haut et Bas-Canada) et d'Américains de la Nouvelle-Angleterre et de New York.. Ces stations atteignirent leur apogée vers la fin du siècle el ceriaines gardent encore aujourd hui une clientele qui leur conserve un reste de prestige. ${ }^{\text {(7) }}$

À la Malbaie et particulièrement dans le village de Pointe-au-Pic, rapporte encore Brière, les villas et les hótels poussèrent comme des champignons à partir des années 1860 et chaque villageois avait aménagé sa maison pour recevoir des pensionnaires, ce qui constituait un revenu d'appoint intéressant.

Dans une étude très documentée sur l'histoire de la villégiature dans Charlevoix, dont La Malbaie est un des joyaux, I'historien Philippe Dubé s'exprime ainsi:

Au $X I X^{r}$ siecle, même si l'on croit fermement que l'esprit oisif dans un corps inactif est l'arelier du diable, on commence à reconnaître les vertus d'un récit prolongé. $L a$ vie routinière des nouveaux citadins fait qu'ils ont grand besoin de briser le rythme des habitudes. Parallelement, la periode des vacances necessite aussi un changement de lieu, un dépaysement, ce qui crée alors un mouvement inhabituel de la ville à la campagne. Le deplacement de ces citadins vers les contrées éloignées des pavés et du browhaha des foules donne naissance à un nouveau mode de vie: la villegiarure. ${ }^{\text {(s) }}$

On croirait en lisant cette citation que nous sommes en plein milieu des années 1980 alors que ces phrases s'appliquent à une petite élite qui possédait fortune et rang social suffisamment élevé pour lui permettre, à cette époque, de goûter aux délices de vacances annuelles dans un décor splendide. Déjà, dans la première moitié du $\mathrm{XIX}^{\mathrm{c}}$ siècle, la grande bourgeoisie américaine avait adopté un certain snobisme à cet égard. Ainsi en était-il, par exemple, de l'élite de Cincinnati:

For example, outside Lowisville some sulphur and mineral springs offered a choice spot for those fleeing the disagreable atmosphere of the cities and McMurtrie observed that its only objectionable feature mas its proximity to Louisville, it being so near, it requires neisher equipage nor the expenses of a jouney to arrive there, things absolutely required to render every place of the kind perfectly a la mode. Cincinnati's elite congregated at Kentucky's Big Bone Lick, where they spent summer months in play and relaxation. ${ }^{19}$

Mais les visiteurs qui viennent à La Malbaiè en cette deuxième moitie du XIX' siétcle non seulement fréquentent-ils un endroit à la mode et forment-ils une société trè̀s fermée mais encore

$L$ idé première de ce nouvel art de wive est de se sentir, pour quelque temps à tout le moins, un arislocrate. En effet, le villegiareur qui s installe dans une villa aux airs parfois précieux d'un chatteau, s'entoure de domestiques et fait ce qui lui plait avec la plus grande delectation. C'est ce qui nows fait dire que l histoire de Charlevoix n'est pas seulement l'hisroire d'un endroit, mais encore celle d'un style de vie umique en Amériqué, ${ }^{(10)}$

Qué faut-il ajouter de plus sinon que c'était effectivement une vie d'aristocrates...

\section{Autour de Montréal}

Dans les environs de Montréal, la villégiature au XIXt siècle n'a pas pris l'allure si particulière de Charlevoix. Cependant, à partir de la deuxième moitié de ce siècle. plusieurs villas ont été construites sur les rives des plans d'eau qui entourent l'íle de Montréal. Au nord, au sud, à l'est et à l'ouest, ces implantations se feront surtout, dans un premier temps, sur le territoire de I'île de Montréal.

Ainsi, a l'est, les petits villages camadiensfrançais de Longue-Pointe et de Pointe-auxTrembles, où plusieurs familles de vieille souche cultivent la terre, verront arriver des notables de Montréal qui, en acquérant quelques fermes, en feront leur grand domaine de villégiature. Ainsi, GeorgesEtienne Cartier, celui qu ion sumomme le pilier de la Confedération, passe l'été à Longue-Pointe dans sa villa au bord du fleuve. Dans le voisinage, on trouve une petite bourgeoisie d'hommes d'affaires et de politiciens en vue; les Symes habitent le domaine $d$ "Elmwood" et les Cuvillier celui de Review Cottage.iil

Vers le nord, sur les rives de la rivière des Prairies, la bourgeoisie locale de Montréal s'installe aussi sur les confins des terres agricoles de l'île Jésus, là oủ sera constituée, à partir du noyau de village existant, la nouvelle municipalité de Laval-desRapides. Un premier pont en bois construit entre l'île Jésus et l'żle de Montréal en 1847 facilitera d'ailleurs cette migration d'estivants.

Dès la seconde moitié du XIXt siècle, les parties riveraines des terres dans l'owest de la ville, ont été détachées des terres agricoles, subdivisées et vendues à des citadins 
fortunés attirés par la proximité de l'eau et la grandeur des terrains, bref par la qualité exceptionnelle du site. Ces demiers s'y construisirent des maisons qui n'ont des résidences d'été que le nom. Ce secteur situé an sud-ouest de la ville constitue l'essentiel de la zone de villégiature a Laval-des-Rapides, zone qui d'ailleurs se transformera rapidement en secteur de résidences permanentes sous l'effet du développement urbain, et qui demeure jusqu'd aujourd'hui le secteur le plus cossu de la ville, ${ }^{(12)}$

L'amélioration des communications entre l'île Jésus et l'île de Montréal - les ponts Viau, Lachapelle et le chemin de fer du nord - amèneront des villégiateurs à s'installer en plus grand nombre, particulièrement à la tête de ces voies.

Au tournant du siècle, quelques-uns de ces estivants décidèrent de s'y installer définitivement. Ce furent les premières transformations de la résidence secondaire en résidence principale. La "banlieurisation" venait de débuter...

Vers l'ouest de l'île de Montréal, autour du lac des Deux-Montagnes et du lac SaintLouis, là où existaient quelques paroisses de paysans francophones, le développement de la résidence secondaire prit très tôt au $\mathrm{XIX}^{\mathrm{c}}$ siècle une orientation ethnique et socio-culturelle très précise. Cette migration saisonnière de citadins montréalais anglo-saxons et fortunés devait marquer l'urbanisation subséquente de cette partie de l'île de Montréal. Aujourd'hui, ce territoire est peuplé en majorité par la bourgeoisie anglophone qui s'y est installée en permanence.

Before considering the continuing development of Pointe-Claire as a town and to understand some of the influences on that growth, it is necessary to deal with the phenomenon of the massive influx of summer residents, largely English-speaking that turned the parish into a playground for a period beginning in 1810 and lasting until World War II... Largely English-speaking it was so not by virtue of excluding the local residents or by discriminating against them in any way, but because the permanent residents lived their own lives and followed their own traditional way of life, while the people of the playground were visitors and transients. ${ }^{(13)}$

C'est en fait à partir de 1856, avec l'arrivée du chemin de fer, que le flux des estivants entre Montréal et l'ouest de l'île commença à grossir; avec la mise en opération d'une deuxième ligne de chemin de fer en 1887 et des premières communications téléphoniques, ce fut l'invasion.

The playground was essentially an english phenomenon. The local population, which was at the beginning of this period $91 \%$ French Canadian and catholic, simply did not participate to any degree to the fun and games, with the exception of regattas. They were not excluded by any rule or prejudice and, in fact, got along very well with the summer people. They simply had other things to do. With the passing of years, though those setrling permanently in Pointe-Claire were largely Englishspeaking, to the point that by 1940 the population was almost equally French and English, no conflicts between the culture arose. The English did their things in their way, in large part to the east of the village, and the French followed their life style, largely in and around the village. ${ }^{(14)}$

Sur la rive sud du Saint-Laurent, devant l'île de Montréal et particulièrement entre les paroisses francophones de Varennes et de Longueuil, s'étalaient dès la première moitié du XIX ${ }^{\mathrm{e}}$ siècle quelques villas de la bourgeoisie anglophone et francophone. Ce mouvement s'accentua en 1860 avec la construction du pont Victoria, premier à enjamber le fleuve. Oeuvre gigantesque et grandiose pour l'époque, ce pont allait permettre un afflux beaucoup plus grand de citadins qui, du coup, envahirent les bords du Richelieu; tout comme d'autres émigraient, au nord, sur les rives de la rivière des Prairies. Ce fut aussi l'expansion du chemin de fer qui facilita le déplacement des citadins sur la rive sud jusqu'aux Cantons de l'Est. Pour les Montréalais à revenus plus modestes, il existait tout de même des excursions organisées par la Compagnie de Navigation de Longueuil qui permettaient, à quelques-uns d'entre eux, de profiter de quelques heures de plein air sur l'île Sainte-Hélène, le grand parc des Montréalais en plein milieu du Saint-Laurent.

En cette fin de $\mathrm{XIX}^{\mathrm{e}}$ siècle, la villégiature privée s'étendit donc principalement sur les rives du fleuve et des rivières ceinturant l'île de Montréal. Elle occupait aussi parfois, mais de façon moins apparente, la campagne environnante. Dans tous les cas, cette villégiature naissante s'est implantée plus facilement grâce aux différentes voies de chemin de fer qui ont transformé le paysage $^{(15)}$. Mais il ne fait pas de doute que "seule une fraction relativement mince des populations urbaines pouvait se permettre la dépense d'un long déplacement en chemin de fer ou le luxe d'une résidence d'été...(16). Les observateurs de l'époque indiquent tous que la clientèle touristique était composée de gens fortunés. L'architecture particulièrement impressionnante de certaines villas laissait transpirer le niveau d'aisance financière de leurs propriétaires. Le géographe Brière rapporte justement la description de N.M. Hinshelwood, auteur d'un guide touristique sur Montréal et ses environs $^{(16)}$. Ce dernier parle d'une longue suite de "magnificient country mansions", le long des rives du fleuve et particulièrement sur le territoire de l'ouest de l'île de Montréal qui était possédé par des "wealthy Montreal business-men".

\section{Conclusion}

Avant même ces terrains de jeux populaires que sont devenus les Laurentides et I'Estrie, on peut donc dire que le SaintLaurent a constitué l'élément essentiel autour duquel s'est organisée la villégiature au Québec pendant plus d'un siècle. La grande aristocratie n'étant plus que souve. nir et l'urbanisation ayant fait son oeuvre autour de Montréal et de Québec, le fleuve a, sous cet aspect, un peu sombré dans l'oubli. Il semble maintenant connaître un regain de vie, par la Côte de Charlevoix, par ses îles redécouvertes, ou tout simplement par ses riverains qui, plus que jamais, ne veulent pas le voir se banaliser à cause de notre inconscience collective. $f$

Notes et références

(1) Philippe Aubé, Deux cents ans de villégiature dans Charlevoix, Québec, Les Presses de l'Uni. versité Laval, 1986, $336 \mathrm{p}$.

(2) J.M. Lemoine, L'album du touriste: archéolo. gie, histoire, littérature, sport, Québec, Augus. tin Côté, 1972, p. 83.

(3) P.-A. Linteau, R. Durocher et J.C. Robert, Histoire du Québec contemporain, Montréal, Boréal Express, vol. 1, De la confédération à la crise (1867-1929), 1979, $660 \mathrm{p}$.

(4) P.A. Linteau et al., op. cit., 1979, pp. 167 à 173.

(5) Roger Brière (1967a), Les grands traits de l'évolution du tourisme au Québec, Bulletin de I'Association des Géographes de l'Amérique Française, no 14, p. 83-95, 1967. Monsieur Briere a aussi livré une très bonne description de ces hauts-lieux de villégiature dans sa these de doc. torat intitulée Géographie du tourisme au Qué. bec, 1967

(6) Roger Brière, Géographie du tourisme au Qué. bec, Montréal, Université de Montréal, Département de Géographie, These de doctorat, p. 23. Citation tirée de: Arthur Buies (1978) Petites chroniques pour 1977, 1967, p. 98.

(7) Roger Brière, Les grands traits..., 1967, p. 30.

(8) Philippe Dubé, op. cit., 1986, p. 103.

(9) Richard C. Wade, The Urban Frontier; the Rise of Western Cities, 1790-1830, Cambridge, Harvard University Press, 1959, p. 313.

(10) Philippe Dubé, op. cit., 1986, p. 103.

(11) CIDEM-Communications, Vers le bout de l'îte, Ville de Montréal, 1985, p. 4 (Pignon sur rue no 12).

(12) Brian R. Matthews, A History of Pointe-Claire, Montréal, Brianor Ltd, 1985, p. 109.

(13) Brian R. Matthews, op. cit., 1985, p. 126.

(14) Voir: Jean-Charles Harvey, Québec, la doulce province, s.l., Chemin de fer national du Canada 1925, 63 p.

(15) Roger Brière, Les grands traits..., 1967, p. 43

(16) N.M. Hinshelwood, Montreal and Vicinity, Montréal, Desbarats and Co., 1903, 153 p. 